\title{
Escort Husimi distributions, Fisher information and nonextensivity
}

\author{
F. Pennini, A. Plastino* \\ Instituto de Física (IFLP), Universidad Nacional de La Plata and Argentina's National Research Council (CONICET), \\ C.C. 727, 1900 La Plata, Argentina
}

Received 3 February 2004; received in revised form 6 April 2004; accepted 8 April 2004

Available online 28 April 2004

Communicated by C.R. Doering

\begin{abstract}
We evaluate generalized information measures constructed with Husimi distributions and connect them with the Wehrl entropy, on the one hand, and with thermal uncertainty relations, on the other one. The concept of escort distribution plays a central role in such a study. A new interpretation concerning the meaning of the nonextensivity index $q$ is thereby provided. A physical lower bound for $q$ is also established, together with a "state equation" for $q$ that transforms the escort-Cramer-Rao bound into a thermal uncertainty relation.
\end{abstract}

(c) 2004 Elsevier B.V. All rights reserved.

PACS: 02.50.-r; 03.65.-w; 89.70.+c

Keywords: Fisher information; Husimi distributions; Escort distributions; Nonextensivity

\section{Introduction}

We will be concerned here with generalizations of two important information-theoretic uncertainty measures: those of Fisher's $(I)[1,2]$ and Wehrl's $(W)$ [3]. The Wehrl entropy verifies the relation $W \geqslant 1$ [4], and this bound represents a strengthened version of the uncertainty principle. A similar case can be made for $I$ $[1,2]$.

In the case of a harmonic oscillator in a thermal state, $W$ coincides with the logarithmic information measure of Shannon's in the high temperature regime. However, it does not vanish at zero temperature, thus

\footnotetext{
* Corresponding author.

E-mail addresses: pennini@fisica.unlp.edu.ar (F. Pennini), plastino@fisica.unlp.edu.ar (A. Plastino).
}

supplying a nontrivial measure of uncertainty due to both thermal and quantum fluctuations [4]. It has been shown in [5] that intriguing connections link $W$ to Fisher's information measure $I$. We will here also generalize these connections.

The above referred to generalizations (indeed, nonextensive extensions [6,7]) of $W$ and $I$, together with their associated uncertainty applications will be shown to shed some light onto the meaning of the nonextensivity parameter $q$. Establishing adequate $q$-criteria still constitutes an open problem for nonextensive thermostatistics, although great progress has been made in deriving from first principles the appropriate $q$-value for special dynamical problems, some of them related to Hamiltonian systems $[6,8]$. These systems are classical ones, though. Our efforts here will be directed, instead, towards quantum systems. 
The Letter is organized as follows. In order to facilitate the reader's task, some preliminary material is presented in Section 2. We start our present quest in Section 3 by first generalizing the concept of Wehrl entropy to an nonextensive environment, obtaining a " $q$-Wehrl" entropy that provides us with a new interpretation for the index $q$ and then studying a Fisher's information measure constructed with what we call "escort Husimi" distributions, which allows us to obtain a physical lower bound to the nonextensivity parameter $q$. Finally, some conclusions are drawn in Section 4.

\section{Background material}

\subsection{Coherent states and Wehrl information}

In [4] the authors discuss quantum-mechanical phase-space distributions expressed in terms of the celebrated coherent states $|z\rangle$ of the harmonic oscillator $(\mathrm{HO})$, whose Hamiltonian operator $\hat{H}$ is given by

$$
\hat{H}_{o}=\hbar \omega\left[\hat{a}^{\dagger} \hat{a}+\frac{1}{2}\right] \text {. }
$$

The coherent states are eigenstates of the destruction operator $\hat{a}$, i.e.,

$$
\begin{aligned}
\hat{a} & =i(2 \hbar \omega m)^{-1 / 2} \hat{p}+(m \omega / 2 \hbar)^{1 / 2} \hat{x}, \\
z & =\frac{1}{2}\left(\frac{x}{\sigma_{x}}+\frac{i p}{\sigma_{p}}\right) \\
& =\left(\frac{m \omega}{2 \hbar}\right)^{1 / 2} x+i(2 \hbar \omega m)^{-1 / 2} p \equiv x^{\prime}+i p^{\prime},
\end{aligned}
$$

with

$x^{\prime}=\frac{x}{2 \sigma_{x}}, \quad p^{\prime}=\frac{p}{2 \sigma_{p}}$,

$\sigma_{x}=\left(\frac{\hbar}{2 m \omega}\right)^{1 / 2}, \quad \sigma_{p}=\left(\frac{\hbar m \omega}{2}\right)^{1 / 2}$,

$\sigma_{x} \sigma_{p}=\hbar / 2$.

Variances $\sigma$ are evaluated for the HO ground state. Coherent states span Hilbert's space, constitute an overcomplete basis and obey the completeness rule [9]

$\int \frac{\mathrm{d}^{2} z}{\pi}|z\rangle\left\langle z\left|=\int \frac{\mathrm{d} p \mathrm{~d} x}{2 \pi \hbar}\right| p, x\right\rangle\langle p, x|=1$, $\mathrm{d}^{2} z=\mathrm{d} \Re(z) \mathrm{d} \Im(z)=\frac{\mathrm{d} p \mathrm{~d} x}{2 \hbar} \equiv \mathrm{d} p^{\prime} \mathrm{d} x^{\prime}$.

Consider now a system characterized by a Hamiltonian $\hat{H}$. Husimi has introduced an important distribution function $[4,10]$

$\mu(p, x)=\langle z|\hat{\rho}| z\rangle$,

associated to the density matrix $\hat{\rho}$ of the system. The function $\mu(p, x)$ is normalized in the fashion

$\int \frac{\mathrm{d} p \mathrm{~d} x}{2 \pi \hbar} \mu(p, x)=\int \frac{\mathrm{d} p^{\prime} \mathrm{d} x^{\prime}}{\pi} \mu\left(p^{\prime}, x^{\prime}\right)=1$,

which makes it evident that $x^{\prime}$ and $p^{\prime}$ are the "natural" $\mu$-variables, of (HO-ground state) variance unity. The distribution $\mu$ is indeed a Wigner distribution smeared over a phase-space region of size $\hbar$ [4]. It is important for our present purposes to remark that the Husimi distribution (HD) is a positive definite one [4]. The HD may be thought of as a "classical" distribution over phase-space [11].

One of the main tenets of Information Theory is that one can associate an information measure to any probability distribution [13]. The Shannon information measure associated to the Husimi distribution is called the Wehrl entropy $W[3]$

$W=-\int \frac{\mathrm{d} p \mathrm{~d} x}{2 \pi \hbar} \mu(p, x) \ln \mu(p, x)$.

As shown by Lieb [12], this special entropic form verifies the inequality

$W \geqslant 1$.

\subsubsection{Canonical Husimi distribution}

Let $\hat{O}$ be an operator relevant for the system's description. The "thermal" mean value of $\hat{O}$ in Gibbs' canonical ensemble is given by [11]

$$
\begin{aligned}
& \langle\hat{O}\rangle=\operatorname{Tr}[\hat{\rho} \hat{O}], \\
& \hat{\rho}=Z^{-1} e^{-\beta \hat{H}}, \quad Z=\operatorname{Tr}\left(e^{-\beta \hat{H}}\right),
\end{aligned}
$$

with $\hat{\rho}$ the system's canonical density matrix, $Z$ the pertinent partition function, and $\beta=1 / k T$, being $T$ the temperature, with $k$ the Boltzmann constant, to be set equal to unity hereafter. 


\subsubsection{HO Husimi distribution}

In the important HO instance $\left(\hat{H} \equiv \hat{H}_{o}\right)$, if we denote with $|n\rangle$ the HO-eigenstates, associated to the eigenvalues $E_{n}=\hbar \omega(n+1 / 2)$, one has [4]

$$
\begin{gathered}
\langle z|\rho| z\rangle=\frac{1}{Z} \sum_{n} e^{-\beta H}|\langle z \mid n\rangle|^{2}, \\
|\langle z \mid n\rangle|^{2}=\frac{|z|^{2 n}}{n !} e^{-|z|^{2}},
\end{gathered}
$$

entailing that

$$
\mu(p, x)=\left(1-e^{-\beta \hbar \omega}\right) e^{-\left(1-e^{-\beta \hbar \omega}\right)|z|^{2}},
$$

or, in terms of the "natural" variables $p^{\prime}$ and $x^{\prime}$

$$
\mu\left(p^{\prime}, x^{\prime}\right)=\left(1-e^{-\beta \hbar \omega}\right) e^{-\left(1-e^{-\beta \hbar \omega}\right)\left[p^{\prime 2}+x^{\prime 2}\right]},
$$

gives the HO Husimi distribution, which, after integration over the phase space, yields an HO Wehrl's entropy

$$
W(\mathrm{HO})=1-\ln \left(1-e^{-\beta \hbar \omega}\right),
$$

that is the Lieb's HO-thermal uncertainty relation [12]. Finally, notice that

$$
\begin{aligned}
e_{|z|}^{2}(\beta, \omega) \equiv & e_{|z|}^{2}=\int \frac{\mathrm{d} p^{\prime} \mathrm{d} x^{\prime}}{\pi} \mu\left(p^{\prime}, x^{\prime}\right)|z|^{2} \\
& \quad-\left[\int \frac{\mathrm{d} p^{\prime} \mathrm{d} x^{\prime}}{\pi} \mu\left(p^{\prime}, x^{\prime}\right)|z|\right]^{2} \\
= & \left\langle|z|^{2}\right\rangle-\langle|z|\rangle^{2} \\
= & \int \frac{\mathrm{d} p^{\prime} \mathrm{d} x^{\prime}}{\pi} \mu\left(p^{\prime}, x^{\prime}\right)\left(p^{\prime 2}+x^{\prime 2}\right) \\
& =\frac{1}{1-e^{-\beta \hbar \omega}},
\end{aligned}
$$

ranges equals unity (Heisenberg's uncertainty lowest limit) for $T=0$ and diverges as $T \rightarrow \infty$, the typical behavior of a thermal uncertainty relation.

\subsection{A brief primer on Fisher's information measure}

A very important information measure is that advanced by R.A. Fisher in the twenties (a detailed study can be found in Refs. [1,2]). Let us consider a system that is specified by a physical parameter $\theta$, while $\mathbf{x}$ is a stochastic variable $\left(\mathbf{x} \in \Re^{N}\right)$ and $f_{\theta}(\mathbf{x})$ the probability density for $\mathbf{x}$, which depends on the parameter $\theta$. An observer makes a measurement of $\mathbf{x}$ and has to best infer $\theta$ from this measurement, calling the resulting estimate $\tilde{\theta}=\tilde{\theta}(\mathbf{x})$. One wonders how well $\theta$ can be determined. Estimation theory [14] asserts that the best possible estimator $\tilde{\theta}(\mathbf{x})$, after a very large number of $\mathbf{x}$-samples is examined, suffers a mean-square error $e^{2}$ from $\theta$ that obeys a relationship involving Fisher's $I$, namely, $I e^{2}=1$, where the Fisher information measure $I$ is of the form

$I(\theta)=\int \mathrm{d} \mathbf{x} f_{\theta}(\mathbf{x})\left\{\frac{\partial \ln f_{\theta}(\mathbf{x})}{\partial \theta}\right\}^{2}$.

This "best" estimator is called the efficient estimator. Any other estimator must have a larger meansquare error. The only proviso to the above result is that all estimators be unbiased, i.e., satisfy $\langle\tilde{\theta}(\mathbf{x})\rangle=\theta$. Thus, Fisher's information measure has a lower bound, in the sense that, no matter what parameter of the system we choose to measure, $I$ has to be larger or equal than the inverse of the mean-square error associated with the concomitant experiment. This result,

$I e^{2} \geqslant 1$,

is referred to as the Cramer-Rao bound [2]. The celebrated Uncertainty Principle of Heisenberg's can be shown to constitute a special instance of (16) [2]. On account of (16) one is in a position to state that $I$ provides us with a positive amount of information [2], as opposite to Shannon's entropy, that measures ignorance [13]. Also, the latter is a global measure, while $I$ is a local one [2]. If $y_{1}, y_{2}, \ldots, y_{n}$ are $n$ relevant parameters of the problem at hand (possibly including $\theta$, but not, of course, $\mathbf{x}$, that is integrated over), we will rewrite (16) in the fashion

$F\left(y_{1}, y_{2}, \ldots, y_{n}\right) \equiv I e^{2} \geqslant 1$.

In the case of the harmonic oscillator, for instance, these parameters are the inverse temperature and the frequency.

A particular $I$-case is of great importance: that of translation families [2,15], i.e., that in which $I$ is a functional of distribution functions (DF) whose form does not change under $\theta$-displacements. These DF are shift-invariant (à la Mach, no absolute origin for $\theta$ ), and for them Fisher's information measure (FIM) adopts the somewhat simpler appearance [2]

$I($ shift-invariant $)=\int \mathrm{d} \mathbf{x} f(\mathbf{x})\left\{\frac{\partial \ln f(\mathbf{x})}{\partial \mathbf{x}}\right\}^{2}$. 
This shift-invariant form of $I$ has encountered many physical applications [2].

We will be concerned below with a special FIMform called the "escort Fisher measure". It was devised, for a nonextensive setting [6,7], by Plastino, Plastino, Miller, and Pennini in $[15,16]$. Let us remind the reader first of all of the useful concept of escort probabilities (see [17] and references therein). Given a normalized, discrete (continuous) probability distribution (PD) $P(i)(f(\mathbf{x}))$, its associated escort PD of order $q$ ( $q$ any real parameter) is defined, for the discrete or continuous case as, respectively, [17],

$P^{(q)}(i)=\frac{P(i)^{q}}{\sum_{i} P(i)^{q}}, \quad$ or

$f^{(q)}(\mathbf{x})=\frac{f(\mathbf{x})^{q}}{\int d \mathbf{x} f(\mathbf{x})^{q}}$.

In the case of complex scenarios involving a PD $P(i)(f(\mathbf{x}))$, it is often the case that the associated escort PDs yield more insights into the concomitant dynamics than the original PD [17]. The escort FIM is then just Fisher's measure expressed as a functional of a escort distribution of order $q$ [15]

$I^{(q)}(\theta)=\int \mathrm{d} \mathbf{x} f_{\theta}^{(q)}(\mathbf{x})\left\{\frac{\partial \ln f_{\theta}^{(q)}(\mathbf{x})}{\partial \theta}\right\}^{2}$,

which obeys, instead of (16), the "escort Cramer-Rao relation" [15]

$F_{q} \equiv q^{2} I^{(q)} e_{q}^{2} \geqslant 1$,

where $e_{q}^{2}$, of course, stands for the mean-square error evaluated with the escort distribution (compare with Eq. (14)).

\subsection{Nonextensive thermostatistics and escort distributions}

Nonextensive thermostatistics is regarded by many authors as a new paradigm for statistical mechanics (see, for instance, $[6,7,18-24]$ and references therein). It is based on Tsallis' nonextensive information measure

$S_{q}=-\int \mathrm{d} \mathbf{x} p(\mathbf{x})^{q} \ln _{q} p(\mathbf{x})$,

where $p(\mathbf{x})$ is a normalized probability density defined for $\mathbf{x} \in \Re^{N}$ and $\ln _{q}(x)=\left(x^{1-q}-1\right) /(1-q)$ is the so-called $q$-logarithmic function [6], a generalization of the standard logarithmic function. The real parameter $q$ is called the index of nonextensivity, the conventional Boltzmann-Gibbs statistics being recovered in the limit $q \rightarrow 1$.

A typical feature of nonextensive thermostatistics is that of employing expectation values constructed with escort PDs. This is, if the quantity $\Delta$ takes the value $\Delta_{i}$ for the event $i$ of probability $P(i)$, then [15]

$\langle\Delta\rangle_{q}=\sum_{i} P^{(q)}(i) \Delta_{i}$,

is to be regarded as the expectation value of $\Delta$ in using the MaxEnt approach [13] in conjunction with $S_{q}$ [21, 24]. Tsallis' nonextensivity index is thereby identified with the order of the underlying escort distribution, as first pointed out in [15]. Summing up, current usage of nonextensive thermostatistics employs three basic ingredients:

(1) Tsallis entropy;

(2) MaxEnt;

(3) $q$-expectation values evaluated with escort distributions.

\subsection{Fisher measure and Husimi distributions}

For the reader's convenience, we summarize first of all results obtained in [5] that involve the shiftinvariant Fisher measure associated to the Husimi probability distribution $\mu(p, x)$. Firstly, remember that Fisher's measure is additive [2]: if $x$ and $p$ are independent variables, $I(p+x)=I(p)+I(x)$, where we denote for $\theta \equiv \tau=(p, x)$ a point in phase-space, so that we face a shift-invariance situation. One defines $z$ in terms of the variables $x$ and $p$, that are scaled by their respective variances (cf. above the definition of $|z\rangle$ ). The ensuing shift-invariant Fisher measure is then [5]

$I($ shift-invariant $) \equiv I_{\tau}=\int \frac{\mathrm{d} p \mathrm{~d} x}{2 \pi \hbar} \mu(p, x) \Gamma$

with

$\Gamma=\sigma_{x}^{2}\left[\frac{\partial \ln \mu(p, x)}{\partial x}\right]^{2}+\sigma_{p}^{2}\left[\frac{\partial \ln \mu(p, x)}{\partial p}\right]^{2}$,

so that we can recast it in the form

$\Gamma=\left(1-e^{-\hbar \beta \omega}\right)^{2}|z|^{2}$. 
The above measure (24) constitutes an estimation tool for location in phase-space. Using here the $\mathrm{HO}$ Husimi $\mu$-expression (5), $I_{\tau}$ adopts the appearance

$$
I_{\tau}(\mathrm{HO})=1-e^{-\beta \hbar \omega},
$$

so that, using (14), we immediately verify that

$F(\beta, \omega) \equiv I_{\tau} e_{|z|}^{2}=1$,

i.e., Cramer-Rao bound is reached. This result is liable to arouse mixed feelings. On the positive side, one sees that efficient estimation is possible at all temperatures, not only at $T=0$. On the debit side, however, we lose in $F(\beta, \omega)$ all temperature-dependence in our à la Cramer Fisher estimation process. We will remedy this situation below by recourse to escort distributions.

Finally, notice also that comparison with Eq. (13) allows us to write [5]

$$
\begin{gathered}
W(\mathrm{HO})=1-\ln \left[I_{\tau}(\mathrm{HO})\right] \\
\Longrightarrow W+\ln \left[I_{\tau}\right]=1,
\end{gathered}
$$

and we regain contextual temperature information, but using both Wehrl's and Fisher's measures. Since the first one manages to do this by itself (cf. Eq. (13)), not too much is gained. What we really want is to obtain such a temperature context by recourse to Fisher's information by itself, without further ado.

\section{The Husimi-Tsallis distribution}

\section{1. q-Wehrl measure}

We will use in what follows the abbreviation $\tau \equiv$ $(p, x)$ and proceed now with the task of generalizing Wehrl's information measure (7) so as to accommodate it to a Tsallis nonextensive environment and thus obtain the concomitant "nonextensive Wehrl entropy" $W^{(q)}$. This is straightforwardly achieved in the fashion

$W^{(q)}=-\int \frac{\mathrm{d} p \mathrm{~d} x}{2 \pi \hbar} \mu(p, x)^{q} \ln _{q} \mu(p, x)$,

the integration process encompassing the whole of phase-space. Explicit evaluation of (30) yields, for the thermal HO,

$W^{(q)}(\mathrm{HO})=q\left\{1+\ln _{q}\left[\left(1-e^{-\beta \hbar \omega}\right)^{-1}\right]\right\}$.
In the limit $q \rightarrow 1$ we have the standard form obtained by Anderson et al. given by Eq. (13), since

$\lim _{q \rightarrow 1} \ln _{q}\left[\left(1-e^{-\beta \hbar \omega}\right)^{-1}\right]=-\ln \left(1-e^{-\beta \hbar \omega}\right)$.

Note that, when the temperature goes to zero $(\beta \rightarrow$ $\infty)$, then

$W^{(q)}(\mathrm{HO}) \rightarrow q$.

This provides us with a new interpretation for Tsallis' nonextensivity index $q$. It is the $q$-Wehrl entropy of an $\mathrm{HO}$ at $T=0$. Additionally, it follows that, in a quantal regime, $q$ cannot be negative. Indeed, according to the most basic tenet of information theory, $W^{(q)}$ represents our ignorance with regards to location in phase-space once we know that the probability distribution for $\tau$ is $\mu(\tau)$ [13]. Obviously, this ignorance-amount cannot be negative. Thus, we obtain a physical lower-bound for $q$

$q \geqslant 0$.

There is more, however. On account of the Lieb bound $W \geqslant 1$ [12], we also get

$q \geqslant 1$.

\subsection{Escort Husimi distributions}

It is now appropriate to introduce an escort $q$-Husimi distribution in the fashion

$\gamma_{q}(p, x)=\frac{\mu(p, x)^{q}}{\int \frac{\mathrm{d} p \mathrm{~d} x}{2 \pi \hbar} \mu(p, x)^{q}}$.

The associated $q$-Husimi-Fisher measure (24) for translation families is then

$I_{\tau}^{(q)}=\int \frac{\mathrm{d} p \mathrm{~d} x}{2 \pi \hbar} \gamma_{q}(p, x) \Gamma_{q}=q^{2} \frac{\int \frac{\mathrm{d} p \mathrm{~d} x}{2 \pi \hbar} \mu(p, x)^{q} \Gamma}{\int \frac{\mathrm{d} p \mathrm{~d} x}{2 \pi \hbar} \mu(p, x)^{q}}$,

since $\Gamma_{q}=q^{2} \Gamma$. Eq. (36) constitutes an escort Husimi estimation tool for location in phase-space.

\subsection{HO application}

\subsubsection{The q-Wehrl-Fisher connection}

Let us connect now the measure $W^{(q)}$ with the shift-invariant Fisher one $I_{\tau}$ through the HO Wehrl 
generalization

$$
W^{(q)}(\mathrm{HO})=q\left\{1-I_{\tau}^{q-1} \ln _{q} I_{\tau}\right\},
$$

where we have used the facts that (i) $\ln _{q}(1 / x)=$ $-x^{q-1} \ln _{q} x, \forall x, \forall q$, and (ii) when the parameter $q$ tends to the unity $W^{(1)} \equiv W$. In the HO-instance we have, (i)

$$
\int \frac{\mathrm{d} p \mathrm{~d} x}{2 \pi \hbar} \mu(p, x)^{q}=\frac{1}{q}\left(1-e^{-\beta \hbar \omega}\right)^{q-1}
$$

so that we can check that, in the limit $q \rightarrow 1$, the function $\mu$ is normalized to unity, and (ii)

$$
\int \frac{\mathrm{d} p \mathrm{~d} x}{2 \pi \hbar} \mu(p, x)^{q}|z|^{2}=\frac{1}{q^{2}}\left(1-e^{-\beta \hbar \omega}\right)^{q-2} .
$$

\subsubsection{Excitation energy}

It is opportune to recall at this point that $|z|^{2}$ is proportional to the excitation energy $E$ [11]

$$
E(z)=\langle z|\hat{H}| z\rangle-\frac{\hbar \omega}{2}=\hbar \omega|z|^{2}=\left\langle z\left|\hbar \omega \hat{a}^{\dagger} \hat{a}\right| z\right\rangle .
$$

Several items are here to be emphasized:

- From (1), (25), (36), and (40)

$$
\begin{aligned}
I_{\tau}^{(q)}(\mathrm{HO}) & =q^{2}\left(1-e^{-\hbar \beta \omega}\right)^{2} \frac{\int \frac{\mathrm{d} p \mathrm{~d} x}{2 \pi \hbar} \mu(p, x)^{q}|z|^{2}}{\int \frac{\mathrm{d} p \mathrm{~d} x}{2 \pi \hbar} \mu(p, x)^{q}} \\
& \equiv\left\langle|z|^{2}\right\rangle_{q},
\end{aligned}
$$

i.e.,

$$
I_{\tau}^{(q)}(\mathrm{HO})=q^{2}\left(1-e^{-\hbar \beta \omega}\right)^{2}\left\langle E / \hbar \omega \|_{q},\right.
$$

which relates the $q$-Fisher information measure to the $\gamma$-thermal mean value of the excitation energy (the $q$ sub-index indicates that we are employing $q$-mean values).

- One finds, by inserting (38) and (39) into (36),

$$
I_{\tau}^{(q)}(\mathrm{HO})=q\left(1-e^{-\beta \hbar \omega}\right)
$$

- Eq. (43) tells us, once again, that $q$ cannot be negative, since it is the ratio of two positivedefinite quantities.

- Since one easily verifies that $e_{q}^{2}$ in Eq. (21) verifies $e_{q}^{2}(\mathrm{HO})=e_{|z|}^{2}(\mathrm{HO}) / q^{2}$, the $q$-CramerRao inequality (16) reads here (cf. Eq. (28))

$$
\begin{aligned}
& F_{q}(\beta, \omega)=q^{2} I_{\tau}^{(q)} e_{q}^{2}=q I_{\tau} e^{2} \equiv q \geqslant 1 \\
& \Longrightarrow q \geqslant 1,
\end{aligned}
$$

as we had previously ascertained following a Wehrl route.

- Comparing Eq. (43) with the information (27) we find the following relation:

$I_{\tau}^{(q)}(\mathrm{HO})=q I_{\tau}(\mathrm{HO})$,

which connects the escort $q$-Fisher information for translation families with the original $(q=1)$ one.

\subsubsection{State equation for $q$}

It has been speculated in the literature that, in some instances, one could face a temperature-dependent $q=q(T)$ nonextensivity index (see, for instance, [24$26]$, and references therein). In such a vein, let us assume that the parameter $q$ is indeed a function of $\beta$. Consideration of the $q$-Cramer-Rao bound (21) leads to the following idea, in order to gain more insight into (44): extremize $q I_{\tau}^{(q)}$ by deriving it with respect to $\beta$ so as to obtain an equation for $q$ as a function of the inverse temperature. Setting

$\frac{\mathrm{d}\left[q I_{\tau}^{(q)}\right]}{\mathrm{d} \beta}=0$,

we obtain a differential equation for $q$

$\frac{\mathrm{d} q}{\mathrm{~d} \beta}\left(e^{\beta \hbar \omega}-1\right)+q \hbar \omega=0$

whose solution is of the form

$q(\beta)=\left(1-e^{-\beta \hbar \omega}\right)^{-1}$,

and thus we find an escort Cramer-Rao relation

$F_{q}(\beta, \omega) \equiv q=\left(1-e^{-\beta \hbar \omega}\right)^{-1}$.

The $q$-Cramer-Rao bound $F_{q}(\beta, \omega)$ becomes (cf. Eq. (14)) a thermal uncertainty relation (TUR) as that of Lieb's [12]. In such a sense one can then argue that, with $q=q(\beta)$ one "optimizes" the information measure in the sense of transforming the associated bound into a TUR. The "equation of state" (47) expresses the nonextensivity index $q$ in terms of the temperature and the frequency. In particular, at zero temperature we see that $q=1$. The present is the first concrete example, as far as we know, of a temperaturedependent $q=q(T)$ nonextensivity index. 


\section{Conclusions}

In this work, by recourse to the concept of escortdistribution, we have performed a study of generalized information measures (GIM) constructed with the Husimi distributions. Investigating the connection of these GIMs with (i) the Wehrl entropy and (ii) thermal uncertainty relations, has allowed us to find:

- Two new interpretations for the nonextensivity index $q$ in terms of

(1) the $q$-Wehrl entropy, and

(2) the $q$-escort Cramer-Rao bound, for the case of the thermal quantum harmonic oscillator.

- A lower bound for $q$, namely, $q=1$, obtained in two different ways.

- A relation between the $q$-Fisher information measure and the thermal mean value of the excitation energy, valid also for $q=1$.

- A "state-equation" that gives $q$ as a function of $T, \omega$ and transforms the $q$-Cramer-Rao bound [15] $F_{q}(\beta, \omega)=q^{2} I_{\tau}^{(q)} e_{q}^{2} \geqslant 1$ into a thermal uncertainty relation.

The HO is, of course, much more than a mere example. Nowadays it is of particular interest for the dynamics of bosonic or fermionic atoms contained in magnetic traps [27-29] as well as for any system that exhibits an equidistant level spacing in the vicinity of the ground state, like nuclei or Luttinger liquids.

\section{References}

[1] B.R. Frieden, B.H. Soffer, Phys. Rev. E 52 (1995) 2274.
[2] B.R. Frieden, Physics from Fisher Information, Cambridge Univ. Press, Cambridge, 1998.

[3] A. Wehrl, Rep. Math. Phys. 16 (1979) 353.

[4] A. Anderson, J.J. Halliwell, Phys. Rev. D 48 (1993) 2753.

[5] F. Pennini, A. Plastino, cond-mat/0312680.

[6] M. Gell-Mann, C. Tsallis (Eds.), Nonextensive Entropy: Interdisciplinary Applications, Oxford Univ. Press, Oxford, 2004.

[7] A. Plastino, A.R. Plastino, Braz. J. Phys. 29 (1999) 50.

[8] V. Latora, A. Rapisarda, C. Tsallis, Physica A 305 (2002) 129, and references therein.

[9] J.R. Klauder, B.S. Skagerstam, Coherent States, World Scientific, Singapore, 1985.

[10] K. Husimi, Proc. Phys. Math. Soc. Jpn. 22 (1940) 264.

[11] J. Schnack, Europhys. Lett. 45 (1999) 647.

[12] E.H. Lieb, Commun. Math. Phys. 62 (1978) 35.

[13] A. Katz, Principles of Statistical Mechanics, Freeman, San Francisco, CA, 1967.

[14] H. Cramer, Mathematical Methods of Statistics, Princeton Univ. Press, Princeton, NJ, 1946.

[15] F. Pennini, A.R. Plastino, A. Plastino, Physica A 258 (1998) 446.

[16] A. Plastino, A.R. Plastino, H.G. Miller, Physica A 235 (1996) 577.

[17] C. Beck, F. Schlögl, Thermodynamics of Chaotic Systems, Cambridge Univ. Press, Cambridge, 1993.

[18] C. Tsallis, Chaos Solitons Fractals 6 (1995) 539; C. Tsallis, Phys. World 10 (1997) 42.

[19] C. Tsallis, Braz. J. Phys. 29 (1999) 1, and references therein.

[20] A.R. Plastino, A. Plastino, Phys. Lett. A 193 (1994) 140.

[21] C. Tsallis, R.S. Mendes, A.R. Plastino, Physica A 261 (1998) 534.

[22] S. Martínez, F. Pennini, A. Plastino, Physica A 295 (2001) 416.

[23] S. Abe, S. Martínez, F. Pennini, A. Plastino, Phys. Lett. A 281 (2001) 126

[24] S. Martínez, F. Nicolás, F. Pennini, A. Plastino, Physica A 286 (2000) 489.

[25] M. Casas, S. Martínez, F. Pennini, A. Plastino, Physica A 305 (2002) 41.

[26] S. Abe, Physica A 269 (1999) 403.

[27] M.H. Anderson, et al., Science 269 (1995) 198.

[28] K.B. Davis, et al., Phys. Rev. Lett. 75 (1995) 3969.

[29] C.C. Bradley, C.A. Sackett, R.G. Hulet, Phys. Rev. Lett. 78 (1997) 985 